\title{
Evolutionary relationships among rice species with AA genome based on SINE insertion analysis
}

\author{
Chaoyang Cheng, Suguru Tsuchimoto, Hisako Ohtsubo \\ and Eiichi Ohtsubo* \\ Institute of Molecular and Cellular Biosciences, the University of Tokyo, \\ Bunkyo-ku, Tokyo 113-0032, Japan
}

(Received 24 May 2002, accepted 2 August 2002)

\begin{abstract}
Previous studies based on morphological and molecular markers indicated that there are two cultivated and five wild rice species within the Oryza genus with the AA genome. In the cultivated rice species, Oryza sativa, a retroposon named $p$-SINE1 has been identified. Some of the $p$-SINE1 members characterized previously showed interspecific insertion polymorphisms in the species with the AA genome. In this study, we identified new $p$-SINE1 members showing interspecific insertion polymorphisms from representative strains of four wild rice species with the AA genome: $O$. barthii, $O$. glumaepatula, $O$. longistaminata, and $O$. meridionalis. Some of these members were present only in strains of one species, whereas the others were present in strains of two or more species. The $p$-SINE1 insertion patterns in the strains of the Asian and African cultivated rice species $O$. sativa and $O$. glaberrima were very similar to those of the Asian and African wild rice species $O$. rufipogon and $O$. barthii, respectively. This is consistent with the previous hypothesis that $O$. sativa and $O$. glaberrima are derived from specific wild rice species. Phylogenetic analysis based on the $p$-SINE1 insertion patterns showed that the strains of each of the five wild rice species formed a cluster. The strains of $O$. longistaminata appear to be distantly related to those of $O$. meridionalis. The strains of these two species appear to be distantly related to those of three other species, $O$. rufipogon, $O$. barthii and $O$. glumaepatula. The latter three species are closely related to one another with $O$. barthii and $O$. glumaepatula being most closely related. A phylogenetic tree including a hypothetical ancestor with all loci empty for $p$-SINE1 insertion showed that the strains of $O$. longistaminata are related most closely to the hypothetical ancestor. This indicates that $O$. longistaminata and $O$. meridionalis diverged early on, whereas the other species diverged relatively recently, and suggests that the Oryza genus with AA genome might have originated in Africa, rather than in Asia.
\end{abstract}

\section{INTRODUCTION}

Rice is one of the most widely cultivated cereal crops in the world. The genus, Oryza, is comprised of two cultivated rice species and twenty-one wild rice species (Vaughan, 1994; Khush, 1997). The wild rice species represent a reservoir of useful genes and have great potential for aiding in the improvement of the cultivated rice variety (Tanksley and McCouch, 1997; Brar and Khush, 1997). The clarification of the genetic relationships of the wild rice species is thus not only theoretically important for understanding the evolution of rice, but also essential for effectively utilizing these wild

Edited by Yoshio Sano

* Corresponding author. E-mail: eohtsubo@ims.u-tokyo.ac.jp resources. Based on cytogenetic analysis (Li et al., 1964; Morinaga, 1964), genomic DNA hybridization (Aggarwal et al, 1997) and DNA sequence analysis of two nuclear genes (Ge et al., 1999), all species in the Oryza genus are classified into six diploid genome types (AA, BB, CC, EE, FF and GG) and four tetraploid genome types (BBCC, CCDD, HHJJ and HHKK) (Vaughan, 1994; Khush, 1997; Ge et al., 1999).

Species with the AA genome have been widely studied because these include the cultivated rice species. The species with the AA genome are classified into two cultivated rice species, $O$. sativa, which is of the Asian origin and cultivated worldwide, and $O$. glaberrima, which is endemic to Africa, as well as five wild rice species, O. rufipogon, $O$. barthii, $O$. longistaminata, $O$. meridionalis and O. glumaepatula. O. rufipogon is distributed throughout 
tropical Asia and Oceania, and has a life cycle ranging from perennial to annual. $O$. barthii (previously called $O$. breviligulata) is typically annual and is distributed throughout west Africa. O. rufipogon and $O$. barthii are thought to be progenitors of $O$. sativa and $O$. glaberrima, respectively (Morishima et al., 1992; Khush, 1997). $O$. longistaminata is another African wild rice species which is perennial and rhizomatous, as well as being partly selfincompatible and allogamous (Chu et al., 1969a; Ghesquiere, 1986). O. meridionalis, the wild rice species in Australia, is annual, whereas O. glumaepatula, the Latin American relative, varies in perenniality. Various levels of hybrid sterility have been observed in interspecific crossings among these species (Chu et al., 1969b; Morishima, 1969; Chu and Oka, 1970; Naredo et al., 1998), which resulted in a reproductive isolation mechanism among species.

The nomenclature and taxonomy of the AA genome species have been in confusion because of rich variation, even within the strains of a species. Isozyme analysis pointed out for the first time that $O$. meridionalis and $O$. longistaminata represent two distinct species, whereas $O$. barthii, $O$. rufipogon and $O$. glumaepatula are closely related (Second, 1985). Results obtained on the basis of nuclear RFLP (Wang et al., 1992, Bautista et al. 2001), RAPD (Ishii et al., 1996, Bautista et al. 2001), AFLP (Aggarwal et al., 1999) and polymorphisms exhibited by other molecular markers (Bautista et al. 2001) are largely consistent with this classification. However, the detailed phylogenetic relationship is still inconsistent between studies.

Short interspersed elements (SINEs) are eukaryotic mobile elements whose transposition uses RNA as an intermediate in a process called retroposition (Maraia, 1995). SINEs range from 70 to $500 \mathrm{bp}$ with each having an internal promoter for RNA polymerase III. Many SINEs appear to be related to tRNAs, whereas a few, such as the primate Alu and rodent B1 family elements, are related to 7SL RNA. As a consequence of retroposition of SINE, descendent members are replicated from the parent member(s) and inserted into the chromosome randomly. The probability that independent insertion occurs at the same locus, or that a SINE is precisely removed, is virtually nil (Nikaido et al., 1999; Shedlock and Okada 2000). The unique and irreversible event of SINE insertion endows them with a perfect character to infer a phylogenetic relationship, which is generally not available for markers based on DNA sequence variation (Shedlock and Okada 2000). Alu insertions have been used to evaluate genetic relationships between human and higher primates (Bailey and Shen, 1993; Bailey and Shen, 1997; Hamdi et al., 1999). Polymorphic insertions of Alu have been used to support the Africa origin of humans (Batzer et al., 1994). SINE insertion analysis has successfully clarified the previously contentious phylogeny of salmonids (Murata et al., 1993), African cichlid tribes (Takahashi et al., 1998), and whales in relation to even-toed ungulates (Shimamura et al., 1997; Nikaido et al., 1999). An insertion analysis of a SINE element has been used to study the relationship of rice (Mochizuki et al., 1993; Hirano et al., 1994) and Brassica species (Tatout et al., 1999).

$p$-SINE1 is the first plant SINE identified in O. sativa and its related rice species (Umeda et al., 1991; Mochizuki et al., 1992). Copy numbers of $p$-SINE1 have been estimated to be about 6500 and 3000 per haploid genome of O. sativa and O. glaberrima, respectively (Motohashi et al., 1997). The enormous volume of SINE amplifications per organism suggests an important evolutionary role for shaping the diversity of Oryza genomes. We have previously identified several $p$-SINE1 members from the $O$. sativa genome, which showed insertion polymorphisms in rice strains with the AA genome (Mochizuki et al., 1993; Hirano et al., 1994). We have recently identified many p-SINE1 members from the O. sativa genome (Motohashi et al., 1997; Cheng et al. unpublished data). Of these, many are present at the respective loci in all the AA genome strains examined, but some show interspecific insertion polymorphism. The polymorphic $p$-SINE1 members include those which form a subfamily (named RA) with three correlated mutations from the $p$-SINE1 consensus sequence and are specifically present in the $O$. sativa-O. rufipogon population (Cheng et al, unpublished data).

In this study, we isolated new $p$-SINE1 members from representative strains with the AA genome of four wild rice species other than $O$. rufipogon, and investigated the presence and absence of these $p$-SINE1 members at the respective loci in the strains of all species with the AA genome. Based on these results, we classified the rice strains and inferred their phylogenetic relationships. We will show that the method used in this study is a powerful new tool for studying the classification of the cultivated and wild rice strains and for inferring the relationships between them.

\section{MATERIALS AND METHODS}

Rice strains used. Rice strains used are shown in Tables 1 and 2. Most of the strains were obtained from Dr. N. Kurata, the Genetic Stock Research Center of National Genetic Institute, Japan. Total genomic DNAs were isolated from these strains with a Nucleon Phytopure kit (Amersham).

Polymerase chain reaction (PCR). PCR was performed with Ex Taq DNA polymerase (Takara) as described previously (Motohashi et al., 1997). PCR products were electrophoresed in a $1.8 \%$ agarose gel. In most cases, when determining the presence or absence of each $p$-SINE1 member by PCR, one unique fragment with or 
Table 1. The presence of $p$-SINE1 members at respective loci in the representative strains of AA genome and other diploid rice species ${ }^{\mathrm{a}}$

\begin{tabular}{|c|c|c|c|c|c|c|c|c|c|c|c|c|}
\hline \multirow{3}{*}{ Member } & \multicolumn{7}{|c|}{ AA } & & \multirow{2}{*}{$\begin{array}{l}\mathrm{BB} \\
\mathrm{Op}\end{array}$} & \multirow{2}{*}{$\begin{array}{l}\mathrm{CC} \\
\mathrm{Oo}\end{array}$} & \multirow{2}{*}{$\begin{array}{c}\mathrm{EE} \\
\mathrm{Oa} \\
\end{array}$} & \multirow{2}{*}{$\begin{array}{c}\text { FF } \\
\text { Obr } \\
\end{array}$} \\
\hline & & & Or & Ogla & Oba & Oglu & $\mathrm{Om}$ & $\mathrm{Ol}$ & & & & \\
\hline & Nippc & R36 & W1954 & W440 & W1581 & W1189 & W2077 & W1624 & W1582 & W0002 & W1538 & W1401 \\
\hline r1 & + & + & + & + & + & + & + & + & I & 1 & I & I \\
\hline r6 & + & + & + & - & + & + & I & - & I & I & I & I \\
\hline r29 & + & + & + & + & + & + & + & - & - & - & - & I \\
\hline r32 & + & + & + & $+^{*}$ & + & + & - & + & - & - & - & - \\
\hline r34 & + & + & - & + & + & + & - & - & I & - & - & - \\
\hline r46 & + & + & + & + & + & + & - & + & - & I & I & I \\
\hline r55 & - & + & + & - & - & - & - & - & - & I & - & I \\
\hline r63 & $+\dagger$ & $+\dagger$ & $+\dagger$ & $+\dagger$ & $+\dagger$ & $+\dagger$ & - & - & I & I & I & I \\
\hline r68 & + & + & + & + & + & + & + & - & - & - & - & I \\
\hline r69 & - & + & + & + & + & + & + & + & I & - & - & I \\
\hline r70 & + & + & + & + & + & + & - & - & - & - & - & I \\
\hline r72 & + & + & + & + & + & + & - & I & I & I & I & I \\
\hline r217 & + & + & + & + & + & + & $+\ddagger$ & + & I & I & I & I \\
\hline r509 & + & + & + & + & + & + & - & + & I & I & I & I \\
\hline r502 & + & + & + & - & - & - & - & - & - & - & - & - \\
\hline r601 & - & - & - & + & + & + & - & - & I & - & - & I \\
\hline r605 & + & + & + & + & + & + & + & + & I & I & - & I \\
\hline r606 & + & + & + & + & + & + & + & - & I & I & I & I \\
\hline r607 & + & + & + & + & + & + & I & I & - & I & I & I \\
\hline r701 & - & - & - & - & - & - & - & \pm & I & - & - & I \\
\hline r702 & - & - & - & - & - & - & - & + & I & - & I & I \\
\hline r703 & - & - & - & - & - & - & + & + & - & - & - & I \\
\hline r705 & - & - & - & - & - & - & - & + & - & - & I & I \\
\hline r706 & + & + & + & + & + & + & + & + & + & + & + & + \\
\hline r707 & - & - & - & - & - & - & + & + & I & I & I & I \\
\hline r708 & + & + & + & + & + & + & + & + & - & - & - & I \\
\hline r709 & + & + & + & + & + & + & + & + & I & I & I & I \\
\hline r801 & - & - & - & - & - & + & - & - & I & I & I & I \\
\hline r803 & + & + & + & + & + & + & - & - & I & - & - & I \\
\hline r804 & + & + & + & + & + & + & + & + & I & I & I & I \\
\hline r806 & - & - & - & + & + & + & - & - & I & I & I & I \\
\hline r807 & - & - & - & + & + & + & - & - & I & - & I & I \\
\hline r808 & + & + & + & + & + & + & + & - & - & - & I & I \\
\hline r901 & - & - & - & - & - & - & + & - & I & I & I & I \\
\hline r902 & - & - & - & - & - & - & + & - & I & I & I & I \\
\hline r903 & + & + & + & + & + & I & + & + & I & + & I & I \\
\hline r904 & - & - & - & - & - & - & + & - & - & - & - & I \\
\hline r905 & - & - & - & - & - & - & + & - & - & - & - & - \\
\hline r906 & - & - & - & - & - & - & + & - & I & - & I & I \\
\hline r907 & - & - & - & - & - & - & + & - & I & - & I & 1 \\
\hline r908 & I & I & / & / & I & I & + & / & I & I & I & I \\
\hline
\end{tabular}

${ }^{a} p$-SINE1 members identified in the cultivated and wild rice genomes are shown in the first column. Members shown in boldface are those of RA subfamily. The wild or cultivated rice strains of the species, O. sativa (Os), O. rufipogon (Or), O. glaberrima (Ogla), O. barthii (Oba), O. glumaepatula (Oglu), O. meridionalis (Om), O. longistaminata (Ol), O. punctata (Op), O. officinalis (Oo), O. australiensis $(\mathrm{Oa})$, and $O$. brachyantha $(\mathrm{Obr})$, are shown at the top together with their genome types, AA, $\mathrm{BB}, \mathrm{CC}, \mathrm{EE}$, and FF. The presence (+) or absence (-) of $p$-SINE1 members at the respective loci was determined by analysis of PCR-amplified fragments (see Materials and Methods). \pm indicates the strain that generates fragments both with and without $p$-SINE1. A slash shows that no PCR fragments was amplified by PCR. The presence of $p$-SINE1 members identified originally in the respective strains are shown by + in boldface. $+{ }^{*}$ and $++^{\dagger}$ indicate $p$-SINE1 members with Tnr 8 and solo-LTR of a retrotransposon (RIRE11), respectively, whereas $+\ddagger$ indicates the r217 member with a LINE (RILN20) in its flanking region.

${ }^{\mathrm{b}}$ Nipponbare. 
Table 2. The distribution of $p-S I N E 1$ members at respective loci in the strains of AA genome species ${ }^{\mathrm{a}}$

\begin{tabular}{|c|c|c|c|c|c|c|c|c|c|c|c|c|c|c|c|c|c|c|c|c|c|c|c|c|c|}
\hline Species & Strain (Ecot & $\frac{t \text { type) }}{}$ & Origin & $\begin{array}{lll}\mathrm{r} 1 \mathrm{r} 2 \\
\end{array}$ & $77 \mathrm{r8}$ & & $502 \mathrm{r}$ & & Fnr8 & & RILN20 & $\begin{array}{ll}\mathrm{r} 901 \\
\end{array}$ & & $\begin{array}{r}\mathrm{r} 904 \\
\end{array}$ & r906 & r701 & $\begin{array}{ll}\mathrm{r} 702 \\
\end{array}$ & & $\begin{array}{ll}\mathbf{r} 703 \\
\end{array}$ & & $77 \mathrm{r} 60$ & & & & \\
\hline O. sativa & onbare & & Japan & + & & & + & & - & - & - & - & - & - & - & - & - & - & - & & & & - & - & \\
\hline & & & Nepal & & & & + & & - & & - & - & - & - & - & - & - & - & - & & - & & - & - & + \\
\hline & & IN & IRRI & & & + & + & + & - & - & - & - & - & - & - & - & - & - & - & & - & & - & - & + \\
\hline & & IN & IR & + & & & + & + & - & - & - & - & - & - & - & - & 1 & - & - & & - & & - & - & + \\
\hline & ang23 & IN & Korea & ++ & & 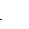 & + & + & - & - & - & - & - & - & - & - & - & - & - & & - & & - & - & + \\
\hline & jing11 & & China & & & & + & + & 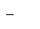 & - & 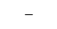 & - & 1 & & - & - & - & & - & & - & & - & - & \\
\hline rufipogon & 54 & $\mathrm{P}$ & China & + & & & + & & - & - & - & - & - & - & - & - & - & - & - & & - & & - & - & \\
\hline & & $\mathrm{P}$ & China & & & & + & + & - & - & - & - & - & - & - & - & - & - & - & & - & & - & - & - \\
\hline & & $\mathrm{P}$ & Indonesia & + & & & + & + & - & - & - & - & - & - & - & - & - & - & - & & - & & - & - & - \\
\hline & & $\mathrm{P}$ & Malaysia & + & & & + & - & - & - & - & - & - & - & - & - & - & - & - & & - & & - & - & + \\
\hline & 05 & $\mathrm{P}$ & India & + & & & + & - & - & - & - & - & - & - & - & - & + & - & - & & - & & - & - & + \\
\hline & W120 & $\mathrm{P}$ & Inc & + & & & + & + & - & - & - & - & - & 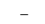 & - & - & - & - & - & & - & & - & - & + \\
\hline & & A & & + & & 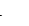 & + & + & - & - & - & - & 1 & - & - & - & - & - & - & & - & & - & - & + \\
\hline & W1690 & A & Thailand & ++ & & & + & + & - & - & - & - & - & - & - & - & - & - & - & & - & & - & - & - \\
\hline & w0107 & A & India & + & & & + & & - & - & - & - & 1 & - & - & - & - & - & - & & - & & - & - & + \\
\hline & 004 & A & India & + & & 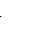 & + & - & - & - & - & - & - & - & - & - & - & - & - & & - & & - & - & + \\
\hline & 07 & м & India & + & & & + & - & - & - & - & - & - & - & - & - & - & - & - & & - & & - & - & + \\
\hline & & $\mathrm{P}, \mathrm{A}$ & India & + & & & + & + & - & - & - & - & - & - & - & 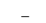 & - & - & - & & - & & - & - & + \\
\hline & & $\mathrm{P}, \mathrm{A}$ & $\mathrm{Ma}$ & + & & & + & - & - & - & - & & & - & - & - & - & & - & & - & & - & - & + \\
\hline & & & & + & & & + & - & - & - & & - & - & - & & & - & - & - & & - & & - & - & + \\
\hline glaberrima & $\mathrm{w}$ & A & $\mathrm{Fr}$ & ++ & & & - & - & + & - & - & - & - & - & - & & - & - & - & & + & & + & + & + \\
\hline & C7599 & A & Nigeria & ++ & & & - & - & + & - & - & - & - & - & - & - & - & - & - & & + & & + & + & + \\
\hline & & A & Sierra Leor & + & & & - & - & + & - & - & - & - & - & - & - & - & - & - & & + & & + & + & + \\
\hline & 38 & A & Guinea & + & & 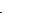 & - & - & + & - & - & - & - & - & - & - & - & - & - & & + & & + & + & + \\
\hline & c & A & Cameroon & + & & & - & - & + & - & 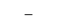 & - & - & - & - & - & - & - & - & & + & & + & + & + \\
\hline & & A & & & & & - & - & + & - & - & 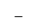 & . & . & & - & - & 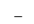 & 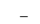 & & + & & + & & + \\
\hline & & A & [bred line] & + & & & - & - & + & - & - & - & - & - & - & - & - & - & - & & + & & + & + & + \\
\hline barthii & & A & & & & & - & - & + & - & & - & - & - & - & & - & - & - & & + & & + & + & + \\
\hline & & A & & + & & & - & - & + & - & & - & - & & & & - & - & - & & + & & + & + & + \\
\hline & & A & & ++ & & 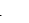 & - & - & + & - & - & - & - & - & - & - & - & - & - & & + & & + & + & + \\
\hline & 73 & A & Ch & + & & & - & - & + & - & - & - & - & - & - & - & - & - & - & & + & & + & + & + \\
\hline & 63 & A & Gambia & + & & & - & - & 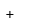 & - & - & - & - & - & - & - & - & - & - & & + & & + & + & + \\
\hline & $\mathrm{w}$ & A & Sierra Leone & + & & & - & - & + & - & - & - & - & - & - & - & - & - & - & & + & & + & + & + \\
\hline & $\mathrm{w}$ & A & Cote-d'Ivoire & + & & & - & - & + & - & - & - & - & - & - & - & - & - & & & + & & + & + & + \\
\hline & & A & & + & & 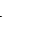 & - & - & + & - & 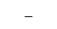 & - & - & - & 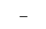 & - & - & - & - & & + & & + & 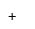 & + \\
\hline & & A & & + & & & - & - & + & - & & - & - & - & - & - & - & - & - & & + & & + & + & + \\
\hline & & A & & & & & - & - & + & & & - & - & & & & - & 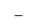 & - & & + & & + & + & + \\
\hline & & A & & + & & & - & - & + & - & & - & - & & & & - & - & - & & + & & + & + & + \\
\hline & & A & & ++ & & & - & - & + & - & & - & - & - & -1 & - & - & - & - & & + & & + & + & + \\
\hline glumaepatula & W1169 & $\mathrm{P}$ & $\mathrm{Cu}$ & + & & & - & - & - & + & - & - & - & - & - & - & I & - & - & & & & + & + & + \\
\hline & & $\mathrm{P}$ & $\mathrm{am}$ & + & & & - & - & - & t & - & - & - & - & - & - & - & - & - & & + & & + & + & + \\
\hline & & & Brazil (Amazon) & + & & & - & - & - & + & - & - & - & - & - & - & I & - & & & + & & + & + & + \\
\hline & & M & $\mathrm{Br}$ & ++ & & & - & - & - & + & - & - & - & - & - & - & - & - & - & & + & & + & + & + \\
\hline & & M & & ++ & & & - & - & - & & & & & & & & - & - & 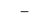 & & & & + & + & + \\
\hline & & M & & ++ & 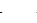 & & - & - & - & + & & & - & & & & & - & 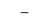 & & & & + & + & + \\
\hline & & M & & + & & & - & - & - & + & & & - & & & & - & - & & & & & + & & + \\
\hline & & м & & + & 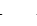 & & - & - & - & + & & & - & & & & & - & & & + & & + & & + \\
\hline & v & м & $\mathrm{Gr}$ & + & & & - & - & - & + & - & - & - & - & - & - & - & - & - & & + & & + & + & + \\
\hline meridionalis & & A & & & & & - & & - & & & & & & & - & - & 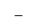 & & & & & - & 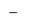 & - \\
\hline & & & $\mathrm{Au}$ & . & & & - & - & - & - & + & + & t & + & + & - & - & - & + & & - & & - & - & - \\
\hline & & A & & & & & - & & & & & & & & & - & - & & & & - & & - & - & - \\
\hline & & A & $A_{t}$ & & & & - & - & & & & & & & & - & - & & & & - & & - & - & - \\
\hline & & A & & + & & & - & - & & & & & & & & & & & & & & & - & - & - \\
\hline & v & A & & + & & & - & - & & & & & & & & & & & & & & & - & & - \\
\hline & & A & & + & & & - & - & & & & & & & & & & - & & & . & & - & & - \\
\hline & & A & & + & & & - & - & & 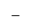 & & & & & & 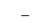 & & - & & & - & & - & & - \\
\hline & & A & Au & & & & - & - & & - & & & & & & - & - & _ & & & - & & - & & - \\
\hline & & A & & + & & & - & - & & & & & & + & & - & & 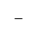 & & & - & & - & - & - \\
\hline & & A & & & & & - & & & & & & & & & & & & & & & & - & & - \\
\hline & & A & & & & & - & & & - & & & & & & - & & & & & 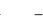 & & - & - & - \\
\hline longistc & & $\mathrm{P}$ & & + & & & - & - & & & & & & & & + & & & & & & & - & - & - \\
\hline & $\mathrm{w}$ & $\mathrm{P}$ & & & & & - & - & & & & & & & & & & & & & & & & & - \\
\hline & & P & & + & & & - & - & & 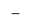 & & & & & & & & & & & 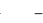 & & - & & - \\
\hline & & P & & & & & - & & & & & & & & & & & & & & & & & & - \\
\hline & & $\mathrm{P}$ & & & & & - & - & & - & & & & & & & & & & & & & - & & - \\
\hline & & $\mathrm{P}$ & $\mathrm{Co}$ & & & & - & & & & & & & & & & & & & & & & & & - \\
\hline & & $\mathrm{P}$ & & & & & - & - & & & & & & & & & & & & & & & - & & - \\
\hline & & P & gascar & & & & - & - & & - & - & & & & & \pm & + & & & & & & - & & - \\
\hline & & $\mathrm{P}$ & & & & & - & - & & & & & & & & & & & & & & & - & & - \\
\hline & & p & & & & & - & & & & & & & & & & & & & & & & & & - \\
\hline & & $\mathrm{P}$ & & & & & - & & & & 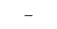 & & & & & & & & & & - & & & & \\
\hline & w070 & & & & & & & & & & & & & & & & & & & & & & & & \\
\hline
\end{tabular}

a The name, ecotype and origin of wild and cultivated rice strains with AA genome were shown in the left columns. Ecotypes of the O. sativa strains, japonica (JA) and indica (IN) are shown. Ecotypes of the other strains, annual (A), perennial (P) and intermediate (M) are also shown. O. rufipogon strains, whose ecotypes are reported as perennial based on one classifier or annual based on another, are indicated by (P, A). Thirty-five $p-S I N E 1$ members or other transposable elements investigated are shown at the top. Two polymorphic $p$-SINE1 members (r905 and r907) in Table 1 were not used for the analysis, because they showed the same polymorphic pattern as r904 and r906. Other features are the same as those shown in Table 1.

without a $p$-SINE1 member was clearly visible in the agarose gel. When the fragments differed in size or when two or more bands were present, the presence or absence of $p$-SINE1 in the fragments was confirmed by Southern hybridization (Mochizuki et al., 1993) or direct sequencing of the PCR products. Primers used for the analysis will be informed upon request.

ADL-PCR (Adaptor-Ligation Based PCR) (Spertini et al., 1999) was performed with Ex Taq DNA polymerase (Takara) as follows: total DNA from W1581 (O. barthii), W1189 (O. glumaepatula), W2077 (O. meridionalis) or W1624 (O. longistaminata) was digested with HindIII, 
which does not cut the p-SINE1 sequence. T4 DNA ligase (New England Biolabs) was used to ligate the digested DNA with the oligonucleotide adaptor. PCR first was performed with a ligated sample as the template using primers that hybridize to the adaptor and the $p$ SINE1 consensus sequence to obtain fragments with the proximal portion of the $p$-SINE1 sequence and its flanking sequence. PCR was then performed with primers that hybridize to the adaptor and a different portion of the $p$-SINE1 sequence. To isolate the fragments with the $p$-SINE1 sequence of the RA subfamily, the 3' ends of the primers used were arranged to match the mutated nucleotides in the $p$-SINE1 sequence of the RA subfamily (see Fig. 1 for primers used). To obtain fragments that included the entire $p$-SINE1 sequence, ADL-PCR was performed with primers that hybridize to the flanking sequence of each identified member and the adaptor.

IPCR (Inverse PCR) was performed as described previously (Tenzen et al., 1994) with total DNA as the template and primers that hybridize to the p-SINE1 consensus sequence. In an attempt to isolate RA $p$ SINE1 members, the 3' ends of two primers for the 1st PCR reaction were arranged to match the mutated nucleotides in the $p$-SINE1 sequence of the RA subfamily (see Fig. 1 for primers used).

Cloning and DNA sequencing. PCR products were cloned into the pGEM-T Easy Vector plasmid (Promega), according to the supplier's instructions. DNA sequencing was performed with a BigDye Terminator kit (PE Bio-

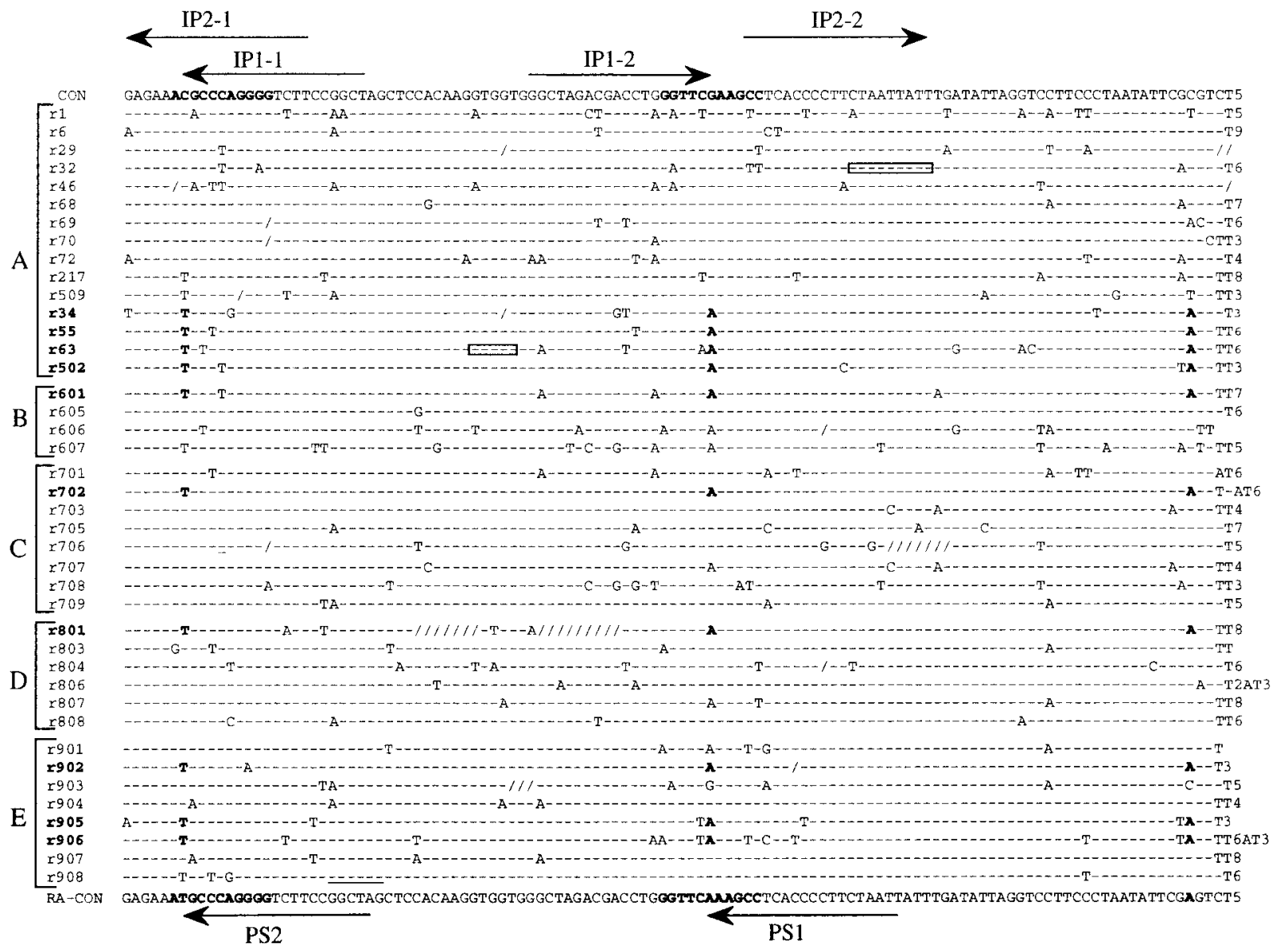

Fig. 1. Nucleotide sequences of the $p$-SINE1 members. Members identified previously in $O$. sativa (A) and new members identified in O. barthii (B), O. longistaminata (C), O. glumaepatula (D) and O. meridionalis (E), are shown. RA-subfamily members are shown in boldface. A consensus sequence (CON) derived from all p-SINE1 members (Motohashi et al., 1997) is shown at the top; a consensus sequence (RA-CON) of RA-subfamily members is shown at bottom. Sequences corresponding to the A- and B-boxes of the pol III promoter are shown in boldface. In each $p$-SINE1 sequence, nucleotides identical to those in the consensus sequence are shown by dashes, and deleted nucleotides by slashes. Underlined nucleotide(s) in r706 and r908 indicates duplication. The three diagnostic mutations of the RA-subfamily members are shown in boldface. Members r32 and r63 respectively have a transposon Tnr8 and a solo LTR of a retrotransposon (RIRE11), whose target site sequences are boxed. Positions of two pairs of primers [IP1-1 and IP1-2 for first PCR and IP2-1 and IP2-2 for second PCR] used to isolate the $p$-SINE1 members by IPCR are shown by the horizontal arrows at the top, while positions of two primers [PS1 and PS2] used to isolate the $p$-SINE1 members by ADL-PCR are shown at the bottom. Note that in attempts to isolate RA-subfamily members, the 3' nucleotides of primers of IP1-1 and IP1-2 as well as PS1 and PS2 are arranged to match the two diagnostic mutations of RA-subfamily members in A box or B box, respectively. 
system) and an ABI PRISM 377 DNA sequencing system. Some PCR product was directly sequenced after electrophoresis in an agarose gel and recovered using SUPREC-01 cartridges (Takara). Nucleotide sequence data reported are available in the DDBJ/EMBL/GenBank databases under the accetion numbers AB088070 - AB088081 and AB088122 - AB088135.

Computer analysis. Primary nucleotide sequences were analyzed with the GENETYX-Mac 10.1 software system. Nucleotide sequence searches made in the DDBJ/Genbank/EMBL databases were performed with the programs, BLAST (Altschul et al., 1990) and SmithWaterman search (Smith and Waterman, 1981). Multiple sequences were aligned using CLUSTAL W (version 1.7) with some manual modifications.

To construct the phylogenetic tree for the various rice strains, the presence or absence of the $p$-SINE1 members at particular loci was organized into a data matrix; the presence of a $p$-SINE1 member at a given locus was coded 1 , and its absence at the same locus coded 0 . Some strains generated two PCR-amplified fragments with or without a $p$-SINE1 member, indicating both the presence and absence of a $p$-SINE1 member, and this case was coded 1 , the presence state. The neighbor-joining (NJ) method was performed using PAUP* $4.0 \mathrm{~b} 8$ software (Swofford, 1998).

\section{RESULTS}

Isolation of new $p$-SINE1 members present in wild rice strains with AA genome. We have previously identified a large number of $p$-SINE1 members present at particular loci in the genomes of three $O$. sativa strains (Nipponbare, IR36 and C5924). Of these, 13 members were found to be polymorphic for insertion in strains of two cultivated and five wild rice species with AA genome (Mochizuki et al., 1993; Motohashi et al., 1997; Cheng et al. unpublished data). The nucleotide sequences of these polymorphic members are shown in Fig. 1, together with two non-polymorphic members, $\mathrm{r} 1$ and $\mathrm{r} 217$. Of these, four members (r34, r55, r63, and r502) belong to the RA subfamily with three diagnostic mutations. Table 1 shows the presence or absence of all $p$-SINE 1 members at the respective loci in representative rice strains from all species with the AA genome, as demonstrated by PCR with a pair of primers that hybridize to the flanking regions of each $p$-SINE1 member.

In this study, we attempted to isolate new polymorphic $p$-SINE1 members from representative strains of the four wild rice species with AA genome, $O$. barthii, O. glumaepatula, $O$. longistaminata and $O$. meridionalis, by inverse PCR (IPCR) with primers matching the $p$-SINE1 consensus sequence. Because a few $p$-SINE1 members belonging to the RA subfamily showed insertion polymorphism in strains of O. sativa/O. rufipogon as well as in some species with the AA genome, we also attempted to isolate more RA-subfamily members by IPCR and adaptor-ligation based PCR (ADL-PCR) from the rice strains used above with primers matching to an RA-subfamily $p$ SINE1 consensus sequence (see the bottom of Fig. 1). Twenty-six members were obtained, and their nucleotide sequences were aligned together with those of the 15 members identified previously (Fig. 1). There were no subfamily members with a set of correlated mutations on the basis of the $p$-SINE1 consensus sequence, except for a few members belonging to the RA subfamily (Fig. 1).

We investigated the presence or absence of all $p$-SINE1 members at the respective loci in representative rice strains from all species with the AA genome, by PCR with a pair of primers that hybridize to the flanking regions of each $p$-SINE1 member. Some $p$-SINE1 members, such as r217 and r804, were present in all the rice strains with the AA genome, similar to member $\mathrm{r} 1$ which has been shown to be present in all the strains of $O$. sativa and $O$. rufipogon (see Table 1). Most of the other members, belonging to either the RA or non-RA subfamily, showed interspecific $p$-SINE1 insertion polymorphisms (Table 1). Most of the members identified in strains of $O$. longistaminata or $O$. meridionalis were present only in those of the same species, whereas most of the members from the other species were present in the strains of two or more species (Table 1).

We also investigated the presence or absence of all $p$ SINE1 members at the respective loci in representative rice strains from four species with non-AA genome (BB, $\mathrm{CC}, \mathrm{EE}$ or FF), as above. We failed to amplify the fragments with most of the members from the strains with non-AA genome (see Table 1), probably because the binding sites of primers used for PCR were lost due to mutations.

Distribution of $p$-SINE1 members among various rice strains with AA genome. To understand relationships among various rice strains with the AA genome, we investigated the distribution of $32 p$-SINE1 members in 72 cultivated and wild rice strains of all species with the AA genome (see Table 2). The three non-polymorphic $p$-SINE1 members, including $\mathrm{r} 1$, were present at their respective loci in all the rice strains (Table 2), clearly indicating that all these strains are derived from a common source. The 29 polymorphic $p$-SINE1 members, however, showed different distribution spectra in the rice strains examined. Some members, such as r801, were present only in the strains of one species, and the others, such as r68, were present in several species (Table 2). Several of these members, such as $\mathrm{r} 34, \mathrm{r} 803, \mathrm{r} 6$ and r69, showed intraspecific polymorphisms in one or two species (Table 2). The five RA members, such as r601, identified in species other than $O$. sativa did not show any 
intraspecific insertion polymorphisms (Table 2).

Some members were specifically present in the strains of each of the wild rice species $(O$. longistaminata, $O$. meridionalis, O. glumaepatula, or O. rufipogon), delineating the strains of each species from others. Note, however, that a few of these members showed insertion polymorphisms among the strains of the species (Table 2). Interestingly, several p-SINE1 members were present in the strains of all the species, except for $O$. longistaminata or $O$. meridionalis, which delineates the strains of these two species from the others (Table 2).

In the strains of $O$. sativa and $O$. rufipogon, which showed a very similar pattern, no particular $p$-SINE1 members could be used to distinguish the strains of $O$. sativa from those of $O$. rufipogon (Table 2). Similarly, in the strains of $O$. glaberrima and $O$. barthii, which showed a very similar pattern, no $p$-SINE1 members could be used to distinguish the strains of $O$. glaberrima from those of $O$. barthii (Table 2).

Notes on transposable elements found within $p$ SINE1 or in regions nearby. In the study of the distribution of $p$-SINE 1 members, three kinds of transposable elements were found to be present within $p$-SINE1 or in regions nearby. One is a DNA type transposable element, called Tnr8, which was inserted in a p-SINE1 member r32 (see Fig. 1 and Table 1; for details, see Cheng et al. 2000). This element was found to be specifically present in r32 in the strains of $O$. barthii and O. glaberrima (Table 2). The second element is a solo-LTR of a retrotransposon, here called RIRE11, which was inserted in another $p$-SINE1 member r63 (see Fig. 1 and Table 1). This element was present in all the strains with $\mathrm{r} 63$ (Table 2). The third element is a LINE-like retrotransposon, called RILN20, which was inserted in the flanking region upstream of another $p$-SINE1 member r217 (see Table 1). This element was specifically present in the strains of $O$. meridionalis (Table 2). These elements supplied additional markers for the classification of rice strains.

Phylogenetic analysis. We carried out a phylogenetic analysis of the 72 cultivated and wild rice strains by constructing a data matrix based on the presence or the absence of $p$-SINE1 members at their respective loci in each rice strain. A phylogenetic tree constructed by the NJ method showed that all the strains can be divided into five groups (Fig. 2). The strains of $O$. longistaminata, $O$.

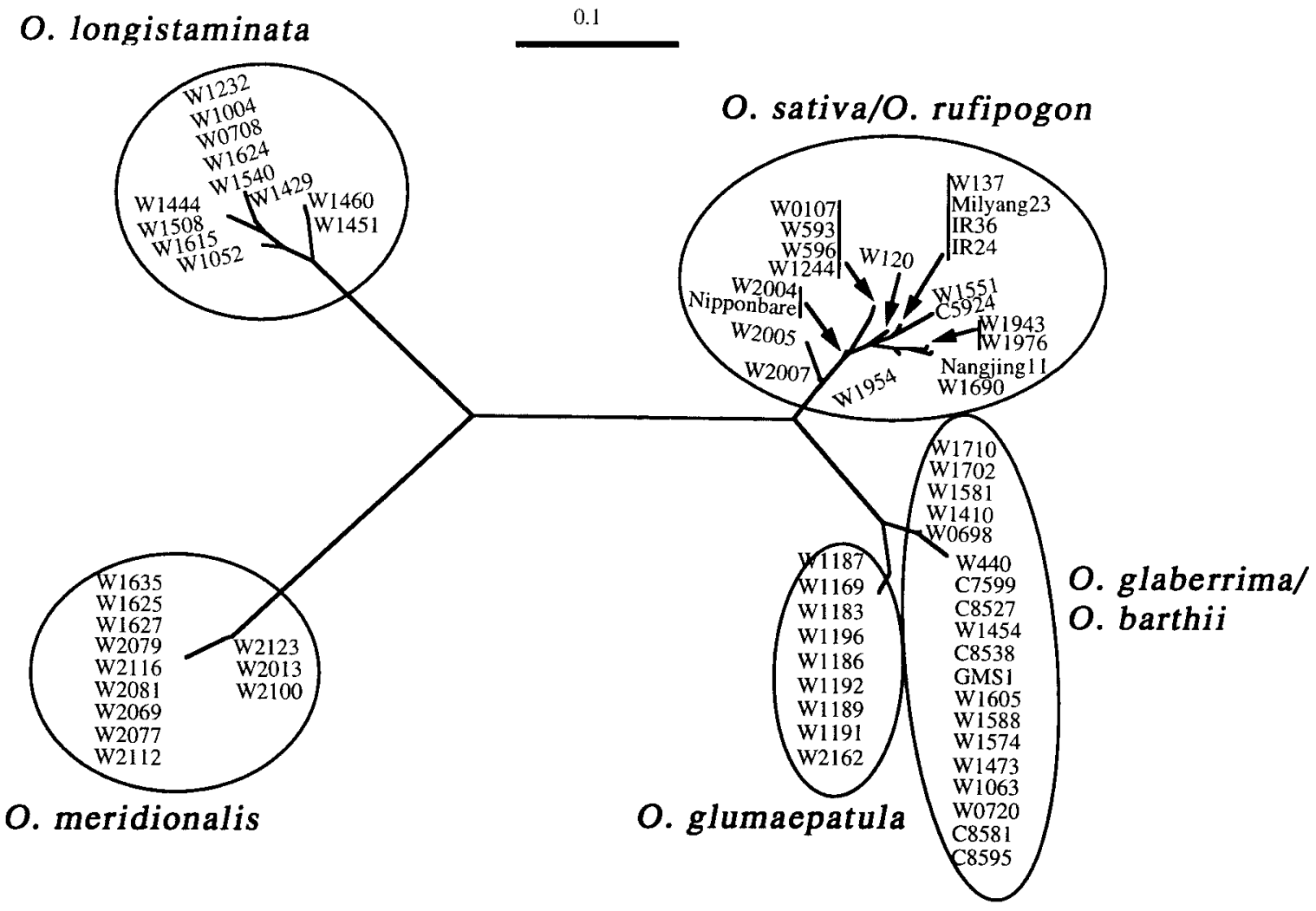

Fig. 2. A phylogenetic tree showing relationships among the 72 strains with the AA genome. All rice strains are listed in Table 2 . The tree was constructed by the NJ method based on the pattern of the presence or absence of $p$-SINE 1 members and three other transposable elements shown in Table 2. All strains were clustered into five groups, corresponding to O. longistaminata, O. meridionalis, O. rufipogon/O. sativa, O. glumaepatula and O. barthii/O. glaberrima. Note that the strains of $O$. rufipogon (or O. barthii) are not distinguishable from those of $O$. sativa (or O. glaberrima) in the tree. The scale bar equals a distance of 0.1 . 
meridionalis, or $O$. glumaepatula were in a cluster forming three distinct groups (Fig. 2). The cultivated rice strains of $O$. sativa, as well as the wild rice strains of $O$. rufipogon, formed the forth group. The cultivated rice strains of $O$. glaberrima and the wild rice strains of $O$. barthii formed the fifth group (Fig. 2). These findings show that the strains of each species with the AA genome can be distinguished almost exclusively by the presence or absence of $p$-SINE1 members and that the present method provides a means of studying the relationships among these rice strains.

The phylogenetic tree shows that the strains of $O$. longistaminata and $O$. meridionalis are distantly related to each other, as well as to those of the other three wild rice species, O. rufipogon, O. barthii and O. glumaepatula (Fig. 2). The strains of the latter three species are closely related to one another, but those of $O$. barthii and $O$. glumaepatula are more closely related. The perennial strains of $O$. longistaminata, O. glumaepatula and $O$. rufipogon and the annual strains of $O$. meridionalis, $O$.

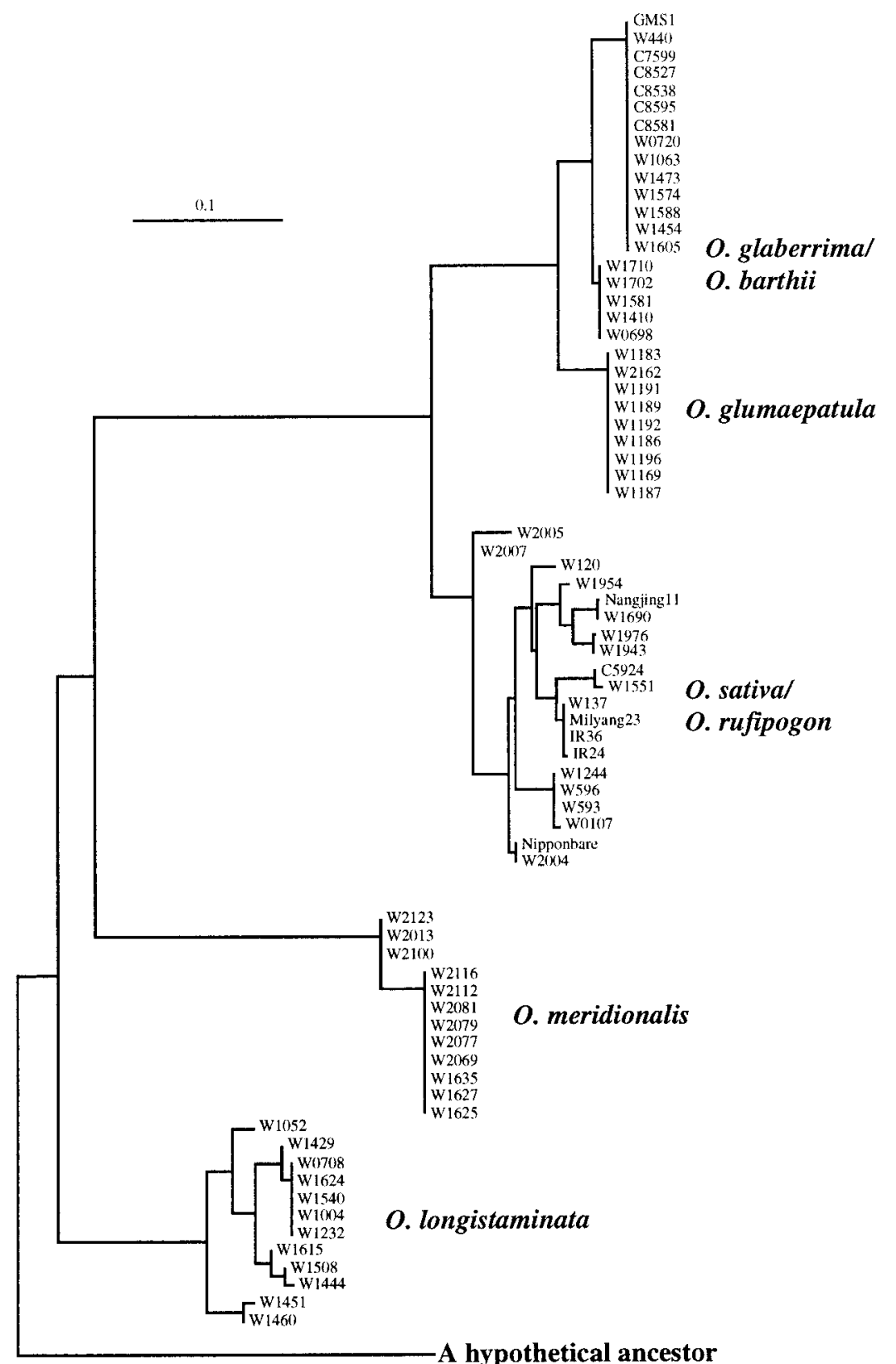

Fig. 3. A phylogenetic tree showing relationships among the rice strains with the AA genome and a hypothetical ancestor. The hypothetical ancestor is defined as the one without all insertions of $p$ SINE1 and other transposable elements. Five groups corresponding to the wild and cultivated rice species are shown. The scale bar equals a distance of 0.1 . 
rufipogon and $O$. barthii are not related to one another (see Table 2 and Fig. 2), showing that there is no correlation between the divergence of species and the divergence of life cycle.

Because all the insertion events could be considered as irreversible events, it is reasonable to assume that the ancestral state would have had no insertion. A hypothetical ancestor with all loci empty for insertions was thus included in the data matrix as an outgroup. The strains of $O$. longistaminata are most closely related to the hypothetical ancestor, with the next closest being $O$. meridionalis and $O$. rufipogon/O. sativa (Fig. 3). The strains of $O$. barthii/O. glaberrima and $O$. glumaepatula are most distantly related to the hypothetical ancestor (Fig. 3).

\section{DISCUSSION}

Classification and relationships of rice strains of species with the AA genome. In this study, we have shown that all strains from each of five wild rice species with the AA genome (O. rufipogon, O. barthii, O. longistaminata, O. meridionalis and O. glumaepatula) exhibit a very similar insertion pattern and thus form a monophyly. This suggests that they evolved independently and is consistent with the conclusion based on the analyses of morphological characters and various molecular markers, such as nuclear RFLP, RAPD, and AFLP, showing that the present method is a powerful alternative tool for classification of rice strains with AA genome.

We have also shown that the Asian cultivated rice strains of $O$. sativa exhibit a very similar insertion pattern to the Asian wild rice strains of $O$. rufipogon, whereas the African cultivated rice strains of $O$. glaberrima exhibit a very similar insertion pattern to the African wild rice strains of $O$. barthii. No $p$-SINE1 members can conclusively distinguish the strains of $O$. sativa (or $O$. glaberrima) from those of $O$. rufipogon (or $O$. barthii). These findings support the view that $O$. sativa and $O$. rufipogon, as well as $O$. glaberrima and $O$. barthii, are actually the same biological species, leading to the hypothesis that strains of $O$. sativa and $O$. glaberrima had been domesticated from those of $O$. rufipogon and $O$. glaberrima, respectively (Morishima et al., 1992; Khush, 1997).

We have also shown that strains of $O$. longistaminata and $O$. meridionalis are distantly related to each other, as well as to those of $O$. rufipogon/O. sativa, O. barthii/O. glaberrima and $O$. glumaepatula. The strains of the latter species are closely related to one another, but those of $O$. barthii/O. glaberrima and $O$. glumaepatula are more closely related. Based on these results, the relationships among species with the AA genome are shown in Fig. 4. Species relationships based on insertion polymorphisms are largely similar to those based on RFLP and

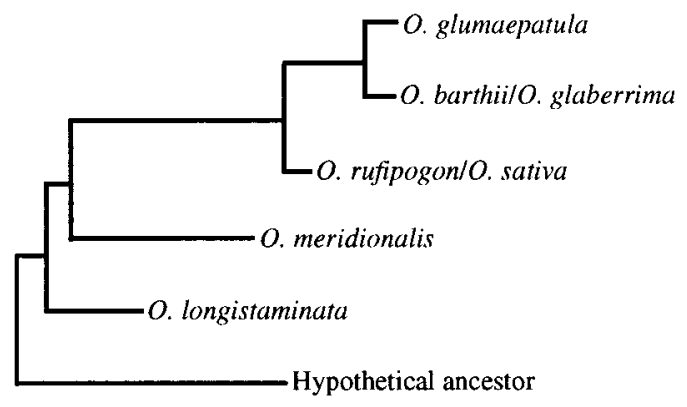

Fig. 4. A phylogenetic tree showing relationships among species with the AA genome and a hypothetical ancestor. See Fig. 3 , from which this figure is derived.

other molecular markers, but the detailed phylogeny is different among these analyses. The relationships among $O$. rufipogon/sativa, $O$. glumaepatula and $O$. barthii/O. glaberrima are the same as those based on nuclear RFLP analysis (Wang et al., 1992; Bautista et al. 2001) and DNA sequence analysis of two nuclear genes (Adh1 and Adh2) (Ge et al., 1999), but are different from those based on the analysis of isozymes (Second, 1985) and other molecular markers, such as AFLP (Aggarwal et al., 1999), RAPD (Ishii et al., 1996), RAPD and NucSSLP (Bautista et al. 2001), which showed that the strains of $O$. glumaepatula or $O$. barthii form a monophyly with those of $O$. rufipogon. Our results suggest that $O$. longistaminata is most distantly related to the other species, which is similar to those obtained by an RFLP analysis (Bautista et al. 2001), but is different from those based on another RFLP (Wang et al., 1992), RAPD (Ishii et al., 1996), and sequence analysis of $A d h$ genes (Ge et al., 1999), which show that $O$. meridionalis is most distantly related to the other species. In this study, we have found at least one species-specific insertion for each species, such as r502 in O. rufipogon and r801 in O. glumaepatula (see Table 2). These species-specific members must have inserted into the genome after their respective host species diverged from their progenitor(s). Also, we have found several $p$-SINE1 members specifically inserted in the strains of two species (see Table 2). These members must have been inserted into the genome of a progenitor. $p$-SINE1 members specific for one or two species supply an unambiguous tag for species identification and relationship analysis.

On the origin of rice species with AA genome. In this study, the ancestral state of $p$-SINE 1 insertion can reasonably be inferred to be absent of insertions, because the $p$-SINE1 insertion is an irreversible event. A Phylogenetic tree including a hypothetical ancestor with all loci empty for $p$-SINE1 insertion showed that the strains of $O$. longistaminata are most closely related to the hypothetical ancestor, with the next closest being $O$. meridionalis and $O$. rufipogon/O. sativa (see Fig. 4). The strains of $O$. 
barthii/O. glaberrima and O. glumaepatula are most distantly related to the hypothetical ancestor (see Fig. 4). These results suggest that $O$. longistaminata diverged earliest in evolutionary time, followed by $O$. meridionalis and $O$. rufipogon, while $O$. barthii and $O$. glumaepatula diverged latest. Our results also suggest that the Oryza species with the AA genome may have originated in Africa, where both $O$. longistaminata and $O$. barthii, which respectively diverged earliest and latest, are sympatric. Although the Oryza genus has been assumed to originate in Asia (Second, 1985; Wang et al., 1992), previous studies which led this assumption had no reliable information concerning for the probable ancestral state of molecular variations, thus it is difficult to confidently determine the root of a phylogenetic tree.

If the AA genome species originated in Africa as suggested above, $O$. longistaminata may have been derived from a progenitor that originated in Africa very early in evolutionary time, whereas $O$. meridionalis from the progenitor migrated to Oceania very early as well. O. rufipogon and $O$. glumaepatula may have been derived from a progenitor(s) that migrated to Asia and South America, respectively, much later than $O$. meridionalis.

$O$. barthii may have been derived from a progenitor that remained in Africa. It has even been suggested, however, that $O$. barthii (annual) evolved from $O$. longistaminata (perennial) based on the paleo-environment evidence that annual strains are generally derived from perennial strains (Chang, 1976). Our results show that strains of $O$. barthii do not form monophyly with those of O. longistaminata (see Fig. 4). It has been demonstrated that there is a differentiation of perennial and annual ecotypes in the wild populations even in a single species $O$. rufipogon (Morishima, 1984). These suggest that $O$. barthii is unlikely to have evolved from $O$. longistaminata. Furthermore, although $O$. longistaminata and $O$. barthii are sympatric in Africa, interspecific introgression does not frequently occur between the two species, as has previously been observed (Chu and Oka, 1970). O. longistaminata is supposed to be one of the two earliest diverged species, whereas $O$. barthii is one of the two latest diverged species, as described above, indicating that the period of the divergence between the two species may be long enough to enable a relatively strict crossing barrier to be developed between the two species.

Genetic introgression and ancestral polymorphism. In this study, we showed that $p$-SINE1 members exhibit different distribution spectrum among the rice strains with the AA genome. Of the $p$-SINE1 members which were present in the strains of a particular species, most do not show intraspecific insertion polymorphism but a few, such as r701 and r901, do (see Table 2 ). This suggests that a few members might not have been fixed in the population of their respective species, because they were amplified relatively recently, but that others might have been fixed, because they were amplified earlier.

Some $p$-SINE1 members were present in the strains of all the species (see Table 2). Of these, a few members, such as r69, showed intraspecific polymorphism in strains of a particular species (see Table 2). The intraspecific polymorphism shown by these members cannot be interpreted as in the case of r701, because they are present in the strains of all species. We assume that insertion polymorphisms in these members might have been derived from a genetic introgression. In the locus with an insertion of member r69, for example, the empty allele in $O$. rufipogon might have been introgressed from a strain of a non-AA genome species with no r69 insertion, probably not long after speciation.

Similarly, in the loci with insertions of r34 and r803, which are present in the strains of $O$. rufipogon, $O$. barthii and O. glumaepatula (see Table 2), the allele with an insertion in $O$. rufipogon might have been introgressed from a strain of a species, such as $O$. barthii or $O$. glumaepatula, with an insertion in the respective locus. Alternatively, the allele with no insertion might have been introgressed from the strains of a species, such as $O$. longistaminata or $O$. meridionalis, with no insertion in the respective locus. The member $\mathrm{r} 6$ showed intraspecific polymorphism in the strains of both $O$. longistaminata and $O$. barthii (see Table 2). More complicated introgression might have occurred in the $r 6$ locus.

The intraspecific polymorphism shown by members, such as r69, may, however, also be explained by the unfixed ancestral polymorphism as discussed previously (Wu, 1991; Hamada et al., 1998). Namely, before these $p$-SINE1 members were fixed in the population of the ancestral species with the AA genome, speciation occurred and the polymorphic SINEs were inherited by and sorted to populations of the respective species. After speciation, these members became fixed, lost, or polymorphic in each derived species as a result of random genetic drift. There is a possibility that such a situation might have occurred, if two or more successive speciation events occur closely in an evolutionary time, and/or if the population size of the ancestral species is too large, as assumed previously (Wu, 1991). The AA genome species are supposed to be rapidly radiated in the Oryza genus (Ge et al., 1999), which makes it possible that ancestral polymorphism might have arisen in the diverged species.

We thank Dr. N. Kurata (National Institute of Genetics, Mishima) for providing the rice strains. This work was supported by a Grant-in-Aid of Scientific Research from the Ministry of Education, Science, Sports and Culture of Japan, and by a grant from the Ministry of Agriculture, Forestry and Fisheries of Japan. 


\section{REFERENCES}

Aggarwal, R. K., Brar, D. S., and Khush, G. S. (1997) Two new genomes in the Oryza complex identified on the basis of molecular divergence analysis using total genomic DNA hybridization. Mol. Gen. Genet. 254, 1-12.

Aggarwal, R. K., Brar, D. S., Nandi, S., Huang, N., and Khush, G. S. (1999) Phylogenetic relationships among Oryza species revealed by AFLP markers. Theor. Appl. Genet. 98, 1320 1328.

Altschul, S. F., Gish, W., Miller, W., Myers, E. W., and Lipman, D. J. (1990) Basic local alignment search tool. J. Mol. Biol. 215, 403-410.

Bailey, A. D., and Shen, C. K. J. (1993) Sequential insertion of Alu family repeats into specific genomic sites of higher primates. Proc. Natl. Acad. Sci. USA 90, 7205-7209.

Bailey, A. D., and Shen, C. K. J. (1997) Molecular origin of the mosaic sequence arrangements of higher primate a-globin duplication units. Proc. Natl. Acad. Sci. USA 94, 51775182 .

Batzer, M. A., Stoneking, M., Alegria-Hartman, M., Bazan, H., Kass, D. H., Shaikh, T. H., Novick, G. E., Ioannou, P. A., Scheer, W., D. Herrera, R. J., and Deininger, P. L. (1994) African origin of human-specific polymorphic Alu insertions. Proc. Natl. Acad. Sci. USA 91, 12288-12292.

Bautista, N. S., Solis, R., Kamijima, O., and Ishii, T. (2001) RAPD, RFLP and SSLP analysis of phylogenetic relationships between cultivated and wild species of rice. Genes Genet. Syst. 76, 71-79.

Brar, D. S., and Khush, G. S. (1997) Alien introgression in rice. Plant Mol. Biol. 35, 35-47.

Chang, T.-T. 1976. The origin, evolution, cultivation, dissemination and diversification of Asian and African rice. Euphytica 25, 425-441.

Cheng, C., Tsuchimoto, S., Ohtsubo, H., and Ohtsubo, E. (2000) Tnr8, a foldback transposable element from rice. Genes Genet. Syst. 75, 327-333.

Chu, Y. E., and Oka, H. I. (1970) The genetic basis of crossing barriers between Oryza perennis subsp. barthii and its related taxa. Evolution 24, 135-144.

Chu, Y. E., Morishima, H. and Oka, H. I. (1969a) Partial selfincompatibility in Oryza perennis subsp. barthii. Jpn. J. Genet. 44, 225-229.

Chu, Y. E., Morishima, H. and Oka, H. I. (1969b) Reproductive barrier distributed in cultivated rice species and their wild relatives. Jpn. J. Genet. 44, 207-223.

Ge, S., Sang, T., Lu, B.-R., and Hong, D.-Y. (1999) Phylogeny of rice genome with emphasis on origins of allotetraploid species. Proc. Natl. Acad. Sci. USA 96, 14400-14405.

Ghesquiere, A. (1986) Evolution of Oryza longistaminata. In: Rice Genetics, pp. 15-25. IRRI, Manila.

Hamada, M., Takasaki, N., Reist, J. D., Decicco, A. L., Goto, A., and Okada, N. (1998) Detection of the ongoing sorting of ancestrally polymorphic SINEs toward fixation or loss in populations of two species of charr during speciation. Genetics 150, 301-311.

Hamdi, H., Nishio, H., Zielinski, R., and Dugaiczyk, A. (1999) Origin and phylogenetic distribution of Alu DNA repeats: irreversible events in the evolution of primates. J. Mol. Biol. 289, 861-871.

Hirano, H., Mochizuki, K., Umeda, M., Ohtsubo, H., Ohtsubo, E., and Sano, Y. (1994) Retrotransposition of a plant SINE into the $w x$ locus during evolution of rice. J. Mol. Evol. 38, 132-137.
Ishii, T., Nakano, T., Maeda, H., and Kamijima, O. (1996) Phylogenetic relationships in A-genome species of rice as revealed by RAPD analysis. Genes Genet. Syst. 71, 195201.

Khush, G. S. (1997) Origin, dispersal, cultivation and variation of rice. Plant Mol. Biol. 35, 25-34.

Li, H. W., Chen, C. C., Wu, H. K., and Lu, K. C. L. (1964) Studies on genetic and cytogenetic evidence for species relationships in the Republic of China. In: Rice Genetics and Cytogenetics, pp. 118-131. IRRI, Elsevier, Amsterdam.

Maraia, R. J. (1995) The Impact of Short Interspersed Elements (SINEs) on the Host Genome. Springer, New York, NY.

Mochizuki, K., Umeda, M., Ohtsubo, H., and Ohtsubo, E. (1992) Characterization of a plant SINE, p-SINE1, in rice genomes. Jpn. J. Genet. 67, 155-166.

Mochizuki, K., Ohtsubo, H., Hirano, H., Sano, Y. , and Ohtsubo, E. (1993) Classification and relationships of rice strains with AA genome by identification of transposable elements at nine loci. Jpn. J. Genet. 68, 205-217.

Morinaga, T. (1964) Cytogenetical investigation on Oryza species.In: Rice Genetics and Cytogenetics, pp. 91-103. IRRI, Elsevier, Amsterdam.

Morishima, H. (1969) Phenetic similarity and phylogenetic relationships among strains of Oryza perennis, estimated by methods of numerical taxonomy. Evolution 23, 429-443

Morishima, H., Sano, Y., and Oka, H. I. (1992) Evolutionary studies in cultivated rice and its wild relatives. Oxford Surveys Evol. Biol. 8, 135-184.

Motohashi, R., Mochizuki, K., Ohtsubo, H., and Ohtsubo, E. (1997) Structures and distribution of $p$-SINE1 members in rice genomes. Theor. Appl. Genet. 95, 359-368.

Murata, S., Takasaki, N., Saitoh, M., and Okada, N. (1993) Determination of the phylogenetic relationships among pacific salmonids by using short interspersed elements (SINEs) as temporal landmarks of evolution. Proc. Natl. Acad. Sci. USA 90, 6995-6999.

Naredo, M. E. B., Juliana, A. B., Lu, B. R., and Jackson, M. T. (1998) Taxonomic status of Oryza glumaepatula Steud. II. Hybridization between new world diploids and AA-genome species from Asia and Australia. Genet. Resour. Crop Evol. 45, 205-214.

Nikaido, M., Rooney, A. P., and Okada, N. (1999) Phylogenetic relationships among cetartiodactyls based on insertions of short and long interspersed elements: hippopotamuses are the closest extant relatives of whales. Proc. Natl. Acad. Sci. USA 96, 10261-10266.

Second, G. (1985) Evolutionary relationships in the Sativa group of Oryza based on isozyme data. Génét. Sél. Evol. 17, 89114.

Shedlock, A. M., and Okada, N. (2000) SINE insertions: powerful tools for molecular systematics. BioEssays 22, 148-160.

Shimamura, M., Yasue, H., Ohshima, K., Abe, H., Kato, H., Kishiro, T., Goto, M., Munechika, I., and Okada, N. (1997) Molecular evidence from retroposons that whales form a clad within even-toed ungulates. Nature 388, 666-670.

Smith, T. F., and Waterman, M. S. (1981) Identification of common molecular subsequences. J. Mol. Biol. 147, 195-197.

Spertini, D., Beliveau, C., and Bellemare, G. (1999) Screening of transgenic plants by amplification of unknown genomic DNA flanking T-DNA. Biotechniques 27, 308-314.

Swofford, D. L. (1998) PAUP*. Phylogenetic Analysis Using Parsimony (*and Other Methods). Version 4. Sinauer, Sunderland, Massachusetts.

Takahashi, K., Terai, Y., Nishida, M., and Okada, N. (1998) A novel family of short interspersed repetitive elements 
(SINEs) from cichilds: the pattern of insertion of SINEs at orthologous loci support the proposed monophyly of four major groups of cichild fishes in Lake Tanganyika. Mol. Biol. Evol. 15, 391-407.

Tanksley, S. D., and McCouch, S. R. (1997) Seed banks and molecular maps: unlocking genetic potential from the wild. Science 277, 1063-1066.

Tatout, C., Warwick, S., Lenoir, A., and Deragon, J. M. (1999) SINE insertions as clade markers for wild crucifers species. Mol. Biol. Evol. 16, 1614-1621.

Tenzen, T., Matsuda, Y., Ohtsubo, H., and Ohtsubo, E. (1994) Transposition of Tnr1 in rice genomes to 5'-PuTAPy-3' sites, duplicating the TA sequence. Mol. Gen. Genet. 245, 441-
448.

Umeda, M., Ohtsubo, H., and Ohtsubo, E. (1991) Diversification of the rice Waxy gene by insertion of mobile DNA elements into introns. Jpn. J. Genet. 66, 569-586.

Vaughan, D. A. 1994. The wild relatives of rice: a genetic resources handbook. International Rice Research Institute, Philippines.

Wang, Z. Y., Second, G., and Tanksley, S. D. (1992) Polymorphism and phylogenetic relationships among species in the genus Oryza as determined by analysis of nuclear RFLPs. Theor. Appl. Genet. 83, 565-581.

Wu C.-I. (1991) Inference of species phylogeny in relation to segregation of ancient polymorphism. Genetics 127, 429-435. 